\title{
Prolegomenon of diagnostic endoscopic ultrasound - from scene dock to stagecraft
}

\author{
I. Borbath, T. Moreels \\ Cliniques universitaires Saint-Luc, Department of Gastroenterology \& Hepatology, Brussels, Belgium.
}

This concise monography (79 pages) on ultrasound and endoscopic ultrasound (EUS) is a remarkably refreshing booklet written by our respected Belgian colleague doctor Vincent De Wilde (1). Yes indeed, the title and subtitle are intriguing. It takes at least two attempts to read and understand it, referring to the eloquence and performing arts of the Ancient Greeks. A prolegomenon is an introductory essay, and that is indeed the most accurate description of this book. The author succeeded to assemble specific topics on (endoscopic) ultrasound, which could be characterized under "everything you always wanted to know, but never dared to ask" or simply "frequently asked questions". In brief, the book reads more like a practical instruction manual rather than the usual fist-thick exhaustive reference books trying to cover all aspects on the subject.

There are 8 chapters, all written in an accessible layman's language, never losing focus on clinical applicability, leaving out in-depth theoretical background information. It explains which buttons on the control panel to turn, push or slide to optimize image quality and to reduce artifacts. The first chapter is an introduction on the basics of physics behind the ultrasound imaging. Although very scientific, it nicely highlights why and how images can be improved. The $2^{\text {nd }}$ chapter explains additional modalities like Doppler, elastography and contrast ultrasound and when and how to use them. Chapter 3 positions the role of EUS in the diagnostic work-up of pancreatic and hepatobiliary conditions, oncological staging and pelvic floor disorders. Chapters 4 and 5 deal with practicalities of endoscope introduction, practical settings of the control panel, safety and adverse events. Chapter 6 is very original in its approach on improving the endosonographist's ergonomics, and about the optimal positioning of the patient and the endoscopy and ultrasound screens. In chapter 7 the standardized EUS report is described in addition to the oncological staging report and the report of dynamic anorectal EUS. The final chapter 8 contains appendices with abbreviations, references and a "visionary and imaginary" philosophical exposition about Plato and Socrates, illustrating the author's personal interest in Ancient Greek culture and philosophy.

The small book format, the to-the-point information with practical tips to improve diagnostic EUS, render the book a helpful tool for daily clinical EUS practice. However, although this EUS prolegomenon aims to be a

\author{
PROLEGOMENON OF \\ DIAGNOSTIC ENDOSCOPIC \\ ULTRASOUND \\ FROM SCENE DOCK TO \\ STAGECRAFT
}

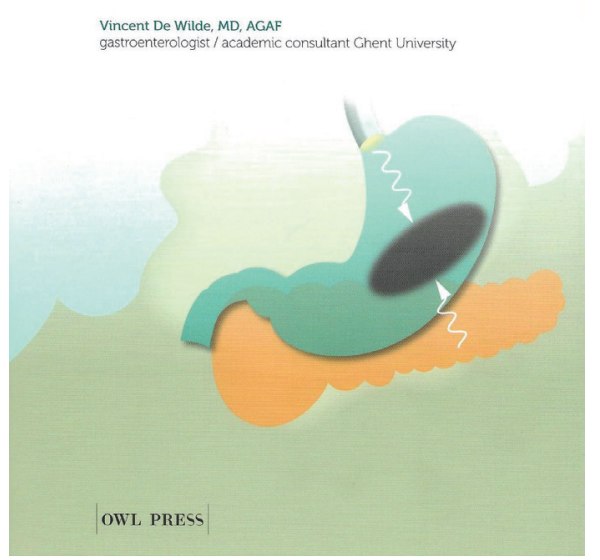

Figure 1. - Front cover of the reviewed book on EUS.

concise instruction manual avoiding far-fetched details, the reviewers missed information on the differential use of radial and linear EUS. And finally, although it is nicely illustrated, the book does not contain a single EUS picture... A reference or link to online available EUS videos would be a useful addition.

The author succeeded to fill the gap between the first introductory graduate EUS courses and the end-ofthe-line reference books. It provides quick answers to pertinent questions endosonographists, both trainees and trainers, are regularly confronted with.

\section{Conflict of interest}

The authors declare no conflict of interest.

\section{Reference}

1. DE WILDE, V. Prolegomenon of Diagnostic Endoscopic Ultrasound - from scene dock to stagecraft. OWL Press, Ghent, Belgium, 2020.

Correspondence to: T. Moreels, Cliniques universitaires Saint-Luc, HépatoGastroentérologie, Avenue Hippocrate 10, 1200 Brussels, Belgium.

E-mail : tom.moreels@uclouvain.be

Submission date : $12 / 12 / 2020$

Acceptance date : 14/12/2020 